\title{
La participation des travailleurs au niveau de l'entreprise et de la société en droit belge
}

Filip Dorssemont et Auriane Lamine

\section{(2) OpenEdition}

1 Journals

Édition électronique

URL : https://journals.openedition.org/rdctss/1603

DOI : $10.4000 /$ rdctss. 1603

ISSN : 2262-9815

Éditeur

Centre de droit comparé du travail et de la sécurité sociale

Édition imprimée

Date de publication : 1 avril 2019

Pagination : 18-39

ISSN : 2117-4350

\section{Référence électronique}

Filip Dorssemont et Auriane Lamine, "La participation des travailleurs au niveau de l'entreprise et de la société en droit belge », Revue de droit comparé du travail et de la sécurité sociale [En ligne], 1 | 2019, mis en ligne le 01 novembre 2021, consulté le 13 novembre 2021. URL : http://

journals.openedition.org/rdctss/1603; DOI : https://doi.org/10.4000/rdctss.1603

\section{(c) (†) $९$}

Revue de droit comparé du travail et de la sécurité sociale est mise à disposition selon les termes de la Licence Creative Commons Attribution - Pas d'Utilisation Commerciale - Pas de Modification 4.0 International. 


\title{
LA PARTICIPATION DES TRAVAILLEURS AU NIVEAU DE L'ENTREPRISE ET DE LA SOCIÉTÉ EN DROIT BELGE
}

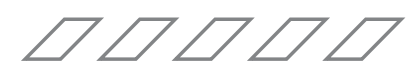

\section{RÉSUMÉ}

La participation des travailleurs au gouvenement des affaires économiques est un objectif que l'on retrouve en Belgique dans les agendas des partis politiques et les revendications syndicales depuis la fin du 19 ème siècle. Cette contribution vise à évaluer si cet objectif se matérialise effectivement dans le droit positif. Une première partie est consacrée à la présentation des mécanismes de participation des travailleurs au gouvernement de l'entreprise ; elle prend le corps du droit du tarvail pour référence. La seconde partie dresse un catalogue raisonné des modes de participation des travailleurs au gouvernement de la société ; elle se fonde sur le droit des sociétés. Dans les deux champs disciplinaires, le bilan apparaît plutôt maigre ».

MOTS CLÉS: Gouvernement de l'entreprise, participation des travailleurs, droit des sociétés, démocratie économique, Belgique.

\begin{abstract}
The participation of employees into the economic governance of the firm has been highlighted as an important objective within the programms of political parties and the demands of trade unions, ever since the end of the 19th century. This contribution seeks to assess whether this objective did materialise within the legal order. The first part of the contribution deals with the instruments of workers' involvement into the economic governance at enterprise level. Labour law is the conceptual framework underlying this analysis. In the second part, a catalogue raisonné of the instruments of worker involvement into corporate governance will be developped. This analysis will be operated on the basis of corporate law. It will appear that Belgian law in both disciplines offers poor prospects to effective participation of employees.
\end{abstract}

KEY WORDS: Corporate Governance, Employee Participation, Company Law, Economic Democracy, Belgium. 


\section{« A LA RECHERCHE DE LA PARTICIPATION PERDUE... »}

"Les signataires du présent projet ont le sentiment de réaliser un premier stade de la démocratie économique. (...) Ils considèrent notamment, que les travailleurs ont un droit indéniable à participer, tant sur le plan général que sur le plan de l'entreprise, non seulement à l'élaboration de la réglementation sociale, mais aussi à la direction de l'économie. Ainsi, ils espèrent résoudre l'antinomie qui s'est affirmée entre la démocratie politique et le pouvoir économique et fondre sous le signe d'une véritable démocratie, les lois de la société politique et de la société économique »"

es déclarations ambitieuses précèdent le texte d'une loi fondamentale "portant organisation de l'économie », adoptée au sortir de la seconde guerre mondiale. Si l'on prend ces quelques lignes au sérieux, il semblerait que le droit belge ait répondu, il y a tout juste septante ans, à une aspiration que les travailleurs avaient déjà exprimée, par l'intermédiaire de leurs représentants, et qui avait été relayée par certains partis politiques, depuis la fin du dix-neuvième siècle : prendre part au gouvernement des entités économiques dans lesquelles ils exercent leurs activités. Pourtant, le terme de participation des travailleurs est quasi-absent du corpus de règles juridiques belge. Il se pourrait ainsi que ces ambitions n'aient pas vraiment été rencontrées.

Le droit belge consacre-t-il effectivement des droits de participation au bénéfice des travailleurs? C'est à cette question complexe que cette contribution tente de répondre.

Celle-ci est structurée en deux volets principaux, consacrés chacun à un champ disciplinaire distinct : le droit du travail (I) et le droit des sociétés (II). II faut d'emblée noter qu'à ces deux corps de règles correspondent le gouvernement de deux entités distinctes : l'entreprise et la société. La participation dont il sera question se distingue déjà par son étendue "rationae loci »: ce n'est pas au gouvernement des mêmes entités que les travailleurs sont appelés à participer, même si celles-ci se recoupent partiellement. Dans ces deux champs du droit, c'est bien sur une notion de participation entendue comme " participation à l'exercice du pouvoir » que nous nous baserons. Nous partons ainsi « à la recherche de la participation perdue ». Cette enquête revêt d'autant plus d'importance que cette aspiration se trouve toujours au cœur des revendications des travailleurs.

La première partie est consacrée à la mise en évidence des formes de participation des travailleurs consacrées par le droit du travail. Après avoir dit quelques mots de cette " entreprise » au gouvernement de laquelle les travailleurs sont amenés à participer, nous présentons les instances représentatives du personnel principales qui se sont vus reconnaître de telles prérogatives. Une tripartition est introduite, qui permet de mieux cerner ces « 50 nuances » de participation : conseil d'entreprise, comité pour la prévention et la protection au travail et délégation syndicale sont tour à tour amenés à partager avec leur employeur et/ ou ses représentants, un pouvoir réglementaire, disciplinaire, et de gestion/direction.

1 Exposé des motifs, loi du 10 septembre 1948 portant organisation de l'économie. 
Les autres modalités d'implication des travailleurs, qui laissent les travailleurs à la marge de l'exercice du pouvoir - c'est-à-dire l'information et la consultation, qui représentent pourtant l'essentiel des compétences des organes susmentionnés - sont délibérément omises de la présentation. Echappent également à cette étude les prérogatives de négociation collective, exercées par les organisations syndicales et les associations d'employeurs. Leur origine se distingue nettement et leur fonctionnement répondent à une autre forme de rationalité de celle des prérogatives que l'on range généralement, en droit du travail, derrière la notion de "participation des travailleurs».

Dans la seconde partie, nous partons à la recherche de traces de participation dans le droit belge des sociétés, entendues ici dans le sens étroit de participation à la direction/ gestion de la société. Après avoir formulé quelques constats sur l'organisation du pouvoir dans les sociétés, nous présentons quelques figures embryonnaires de participation des travailleurs : la participation financière, la participation dans la société à finalité sociale, la figure du dirigeant-salarié, le cas emblématique de la Société nationale des chemins de fer belge. La conclusion dresse quelques constats critiques sur l'état actuel de la participation des travailleurs au gouvernement des organisations économiques au service desquelles ils sont employés.

\section{I - LA PARTICIPATION DES TRAVAILLEURS AU NIVEAU DE L'ENTREPRISE EN DROIT (DU TRAVAIL) BELGE}

\section{A - NOTION D'ENTREPRISE, CONSTITUTION ET COMPÉTENCES DU CONSEIL D'ENTREPRISE}

Il n'est pas aisé de déterminer si le droit du travail belge consacre une idée de " participation » au niveau de l'entreprise. Il y a d'abord l'épineuse question de la définition de l'entreprise en droit du travail belge et, ensuite, celle de la portée de la notion de participation. Néanmoins, la distinction entre le droit du travail et le droit des sociétés offre une clé de lecture utile. La notion d'entreprise en droit du travail et celle de société au sens du droit des sociétés doivent être distinguées.

La société, en tant que personne morale, dirige une ou plusieurs entreprises, le périmètre de cette «entreprise » ou de ces entreprises ne correspond pas nécessairement à celui de la société. La notion d'entreprise, en droit du travail belge, désigne une organisation impliquant à la fois des travailleurs et le «chef d'entreprise ». La notion de société n'implique pas in se et per se que cette société ait effectivement conclu des contrats de travail avec des travailleurs. Le «substrat » en termes de ressources humaines de l'entreprise peut impliquer des travailleurs ayant conclu des contrats de travail avec une panoplie de sociétés prises individuellement. Le concept d'entreprise en droit du travail belge a été défini dans la loi portant organisation de l'économie en tant qu'unité technique d'exploitation². Cette unité

2 Article 14 § 1, Loi du 10 septembre 1948 portant organisation de l'économie, M.B., 27 septembre 1948. 
technique est définie à partir de critères économiques et sociaux. En cas de doute, ces derniers prévalent ${ }^{3}$. La notion d'entreprise se confond ainsi avec celle d'établissement ${ }^{4}$.

Cette confusion critiquable, sur le plan conceptuel, se comprend dans un système qui ne connaît que le conseil dit d'entreprise et qui méconnaît le comité d'établissement. Sur le plan juridique cette confusion a posé et continue à poser problème à la lumière de la distinction entre entreprise et établissement opérée par la Directive cadre 2002/145. Cette approche de la notion d'entreprise permet de surmonter le risque de la circonvention du seuil élevé requis pour l'institution des conseils d'entreprise (100 travailleurs) par la création d'une panoplie de sociétés actives au sein du périmètre de l'entreprise. Toutefois, si une entreprise occupe " en tant qu'entité juridique au moins 50 travailleurs » et « quel que soit le nombre de travailleurs occupés dans chacun de ses sièges ${ }^{6}$, elle est obligée d'instituer un conseil d'entreprise. Dans ce cas, l'entreprise est appréhendée comme la personne juridique.

Cette analyse de la notion d'entreprise, par la force des choses polysémique, n'épuise pas la question de la portée de la définition de participation. Dans certains pays, des Constitutions «modernes » et adoptées ab ovo ont conditionné et balisé le discours sur la participation des travailleurs. L'article 165 de la Weimar Verfassung (1919), évoquant l'idée que les ouvriers et les employés étaient " gleichberechtigt in Gemeinschaft mit den Unternehmern an der Regelung der Lohn- und Arbeitsbedingungen sowie an der gesamten wirtschaftlichen Entwicklung der produktiven Kräfte mitzuwirken », a préfiguré la notion de participation, bien présente dans le préambule de la Constitution française de $1946^{7}$ et dans l'article 46 de la Constitution italienne de $1948^{8}$.

3 Art. 14, loi du 20 septembre 1948.

4 F. Dorssemont, "De impact van de Kaderrichtlijn 2002/14.EG betreffende de informatie en raadpleging in de "onderneming " : verbreding en verdieping", in P. Humblet et J. Vanthournout, De sociale verkiezingen doorgelicht, Antwerpen, Intersentia, 2007, p.174.

5 Directive 2002/14/CE du Parlement européen et du Conseil du 11 mars 2002 établissant un cadre général relatif à l'information et la consultation des travailleurs dans la Communauté européenne Déclaration conjointe du Parlement européen, du Conseil et de la Commission sur la représentation des travailleurs, J.O., L 80, 23.3.2002, p. 29-34

6 Toujours l'article 14.

7 «Tout travailleur participe, par l'intermédiaire de ses délégués, à la détermination collective des conditions de travail ainsi qu'à la gestion des entreprises ».

8 " Ai fini della elevazione economica e sociale del lavoro e in armonia con le esigenze della produzione, la Repubblica riconosce il diritto dei lavoratori a collaborare, nei modi e nei limiti stabiliti dalle leggi, alla gestione delle aziende » (Art. 46). 


\section{B - LES AMBIGUIITÉS DE LA NOTION DE « PARTICIPATION » EN DROIT DU TRAVAIL BELGE}

Au lendemain de la Deuxième guerre mondiale, la Constitution belge est restée à l'abri de telles modifications. Dans le projet d'accord de solidarité sociale 1944, c'est bien le terme " collaboration paritaire » (au sein et au niveau de l'entreprise) qui a été privilégié et non celui de participation". Quant au domaine pointu des "services sociaux institués par les entreprises pour le bien-être du personnel (logements, transports, jardinage, achats en commun, réfectoires, sports, délassements, vacances, enseignement professionnel ou général, bibliothèques, consultations et soins médicaux, etc.) », le texte privilégie la "gestion autonome des travailleurs », sinon «l'administration avec leur concours ».

Malgré la consécration tardive (1993) des droits économiques, sociaux et culturels, le constituant belge n'a pas pu ou voulu profiter de cet acquis historique italo-franco-allemand. L'assemblée constituante s'est limitée à la consécration de la modalité la plus classique de l'implication des travailleurs, c'est-à-dire le droit à l'information et la consultation. Il est d'ailleurs étonnant que les projets de révision de la Constitution qui ont été déposés en vue d'inscrire les droits économiques, sociaux et culturels ne font jamais référence à l'existence d'un droit à la participation.

Que faut-il donc entendre par participation, en droit du travail ? Il ne s'agit donc pas, à notre estime, de déterminer si les travailleurs font partie ou non de l'entreprise. Le simple fait que la loi portant organisation de l'économie suggère une conception aussi ambiguë que l'idée de communauté de travail ${ }^{10}$, implique que ses travailleurs en font partie. L'objet de la participation ne se définit donc pas en fonction d'une organisation, mais en fonction des pouvoirs qui y sont exercés. Autrement dit, elle se conçoit comme une forme de démocratisation de cette organisation.

A l'instar du trias politica propre à l'ordre juridique étatique, il convient de distinguer le pouvoir réglementaire du pouvoir disciplinaire et du pouvoir de "gestion » ou de " direction » exercés dans l'entreprise ${ }^{11}$. Pour que le mot participation à l'exercice du pouvoir ait un sens quelconque, nous ne prendrons pas en considération les modes d'implication qui laissent les travailleurs ou leurs représentants à la marge du processus de l'exercice de ces pouvoirs. Le législateur ne se sert d'ailleurs que rarement du vocable « participation». La seule référence à la notion de participation dans le contexte des lois régissant les relations professionnelles se situe dans la loi relative au bien-être des travailleurs, dont l'article 53 dispose que les travailleurs « participent directement au traitement des questions

9 Ce texte qui n’a jamais été adopté par le législateur a été publié dans la revue officielle du Ministère de l'Emploi (la Revue du Travail). Bien que ce texte ne reflète que la vision d'un comité de sages proche des milieux syndicaux patronaux et de l'administration, il constitue un programme d'action législative qui a été progressivement mise en œuvre au lendemain de la Deuxième guerre mondiale.

10 Ainsi l'idée de collaboration est bien présente dans la loi portant organisation de l'économie, citée ci-dessus. L'entreprise y est évoquée comme une structure dirigée par "le chef d'entreprise ». L'article 15 dispose que le conseil d'entreprise doit « examiner toutes mesures propres à favoriser le développement de l'esprit de collaboration entre le chef d'entreprise et son personnel ».

11 Voir à ce propos la thèse de P. Humblet, Degezagsuitoefening door de werkgever, Antwerpen, Kluwer rechtswetenschappen, 1994, 420p. et F. Dorssemont, "De onderneming: arbeidsgemeenschap of rechtsorde? Omtrent de mogelijkheidsvoorwaarden, de grondslagen, de beperkingen, de demonen, de gevaren en de voordelen van de institutionele leer, casu quo de institutionele leerstelsels », Tijdschrift voor Privaatrecht, 2003, pp. 1313-1412. 
relatives au bien-être des travailleurs » dans les entreprises où il n'existe ni comité pour la prévention et la protection au travail ni délégation syndicale (voy. infra) ${ }^{12}$. Paradoxalement, la figure de la participation est donc utilisée dès le moment où les instances représentatives du personnel sont absentes. Elle a trait, non pas à la portée de l'implication, mais à ses modalités formelles.

Ce que nous envisageons par "participation " est donc une forme de " codétermination » dans l'exercice de ces pouvoirs. La notion de co-détermination nous semble avisée lorsque le chef d'entreprise est incapable de procéder unilatéralement à des décisions ou à adopter des règlements sans l'approbation d'une instance représentative du personnel. Lorsque cette instance est « mixte», l'expression de co-détermination pourrait être malheureuse. Un pouvoir de détermination est ainsi attribué à une instance mixte (le conseil d'entreprise ou le comité pour la prévention et la protection) qui confine au constat que ni le chef d'entreprise ni les représentants des travailleurs ne peuvent décider isolément. Tandis que la co-détermination se traduit par un pouvoir d'adopter une décision ou un règlement, la co-gestion se traduit par un pouvoir plus structurel de gérer au quotidien une entreprise. Ce pouvoir se traduit bien entendu par autant de décisions individuelles ou réglementaires que possible, ainsi que par le pouvoir de dessiner une politique ou stratégie sous-jacente. Les pouvoirs de participation ne sont pas complétement absents dans le droit des relations collectives belge, mais ils sont bien cantonnés dans le domaine dit «social ». Le législateur belge suggère ainsi une étanchéité entre une sphère sociale et une sphère économique et financière.

\section{C - DeS ORgANES ET DES COMPÉTENCES}

Le conseil d'entreprise n'est pas la seule instance représentative des travailleurs. La loi relative au bien-être des travailleurs (op. cit., mise en exécution par un Arrêté Royal (AR) du 3 Mai 1999) a institué le comité pour la prévention et la protection au travail. Ses attributions sont définies par l'article 65 de la loi ainsi qu'aux articles 2 à 13 de l'AR du 3 mai 1999 relatif aux missions et au fonctionnement des comités pour la prévention et la protection au travail (CPPT) ${ }^{13}$. II s'agit d'une instance de concertation ayant une mission spécialisée dans le domaine du bien-être. La notion de bien-être dépasse la référence plus traditionnelle de protection de la santé et de la sécurité.

Une convention collective $n^{\circ} 5$ conclue au sein du Conseil national du travail prévoit aussi une délégation syndicale (DS) qui représente les travailleurs syndiqués dans l'entreprise ${ }^{14}$. L'expression française de représentant du syndicat était inacceptable pour le monde patronal belge. La délégation syndicale a pourtant la capacité de représenter l'ensemble des travailleurs lorsqu'elle est impliquée dans la négociation des conventions collectives d'entreprise. Ces conventions doivent cependant être signées par une autre personne, qui représente l'organisation représentative des travailleurs ${ }^{15}$. Contrairement

12 Loi du 4 août 1996 relative au bien-être des travailleurs lors de l'exécution de leur travail, M.B., 18 septembre 1996, p. 24309.

13 M.B., 10 juillet 1999.

14 CCT n 5 du 24 mai 1971 concernant le statut des délégations syndicales du personnel des entreprises (...), http://www.cnt-nar.be/CCT-COORD/cct-005.pdf

15 Sur cette notion: Art. 3, loi du 5 décembre 1968 sur les conventions collectives de travail et les commissions paritaires, M.B., 15 janvier 1969, p.267. 
aux CE et au CPPT, qui sont composés de représentants des travailleurs et de l'employeur, la délégation syndicale n'est pas une instance mixte. Ces trois acteurs - conseil d'entreprise, le comité pour la prévention et la protection au travail et la délégation syndicale - sont amenés, en droit belge, à prendre une part dans l'exercice de ces trois pouvoirs - réglementaire (1), disciplinaire (2), et de gestion (3).

\section{1 - Le pouvoir réglementaire}

Le pouvoir réglementaire reconnu aux travailleurs, par l'intermédiaire de leurs représentants, concerne essentiellement l'élaboration et la modification du règlement de travail de l'entreprise ${ }^{16}$.

La (méta) réglementation du "règlement de travail ", appelé autrefois "règlement d'atelier ", est antérieure à l'introduction de la figure juridique du contrat de travail. Tandis que le contrat de travail, qui se distingue du contrat de louage de services, a été introduit par les lois de 1900 et de 1922, depuis 1896, la loi sur les règlements d'atelier (1896) tend à baliser le pouvoir réglementaire patronal. Dès le début, cette loi a également circonscrit le pouvoir disciplinaire. Depuis l'adoption de la loi portant organisation de l'économie (1948), le chef d'entreprise a été destitué de son pouvoir réglementaire. Cette loi introduit une antinomie en disposant que le conseil d'entreprise est compétent pour élaborer et modifier le "règlement d'atelier ", contrairement à la loi sur les règlements d'atelier qui confiait cette tâche au chef d'entreprise ${ }^{17}$.

Une nouvelle loi a substitué le règlement de travail au règlement d'atelier et confirmé le principe qu'il incombe au conseil d'entreprise d'adopter le règlement d'atelier ${ }^{18}$. Ce n'est qu'à défaut de conseil d'entreprise que le chef d'entreprise pourra adopter, seul, le règlement. Toutefois, contrairement à ce que prévoyait la loi antérieure, le chef d'entreprise n'est pas juridiquement capable d'imposer sa volonté. II doit permettre aux travailleurs de s'exprimer. En cas de contestation exprimée par les travailleurs ou par les délégués élus, le chef d'entreprise doit passer obligatoirement par une phase de médiation et de conciliation. Il doit faire appel à l'inspection sociale qui devra essayer d'aplanir le différend. Si cette tentative de conciliation échoue, une deuxième phase de médiation et de conciliation doit être menée au sein de la commission paritaire ${ }^{19}$. En cas d'échec, cette commission se transformera afin d'exercer un rôle tout à fait inhabituel, celui d'arbitre, dont la décision sera obligatoire.

La loi portant organisation de l'économie évoque en outre que le conseil d'entreprise est compétent afin de déterminer les critères généraux à appliquer par l'employeur, en cas de licenciement ou de réembauchage résultant de circonstances d'ordre économique

16 Voir La loi du 12 avril 1965 instituant le règlement du travail, M.B., 5 mai 1965, p. 5064

17 Article 15 d) Loi portant organisation de l'économie, op. cit., et article 4 de la loi du 12 avril 1965 instituant le règlement du travail, op. cit.

18 La Loi du 12 avril 1965 instituant le règlement du travail, op. cit.

19 II s'agit de l'organe, composé paritairement de représentants des organisations représentatives des travailleurs et des employeurs, au sein duquel sont négociées les conventions collectives de branche (Loi du 5 décembre 1968, op. cit) 
ou technique (par exemple, critères relatifs à l'âge, à l'ancienneté, aux charges de famille), étant entendu que la décision de licencier appartient à l'employeur ${ }^{20}$.

\section{2 - Le pouvoir disciplinaire}

Le pouvoir disciplinaire reste l'apanage exclusif de l'employeur. Aucun représentant des travailleurs ne peut y participer. Rien ne permet de dire que le conseil d'entreprise devrait être informé ou consulté. En effet, ses compétences ne concernent pas les questions d'ordre individuel. Quant à la délégation syndicale, l'article 13 de la Convention Collective $n^{\circ} 5$ prévoit que la délégation a le droit « d'être reçue à l'occasion de tout litige ou différend de caractère individuel qui n'aurait pu être résolu par la voie hiérarchique ». Il est donc évident que le travailleur qui fait l'objet d'une procédure disciplinaire peut se faire assister par un membre de la délégation syndicale. Aucune disposition ne prévoit malheureusement que le travailleur qui fait l'objet d'une procédure disciplinaire a le droit d'être entendu, ce qui, dans la pratique, peut compromettre l'effectivité du droit reconnu à la délégation syndicale.

L'exercice du pouvoir disciplinaire est fortement balisé par un instrument qui sera toujours le fruit de la concertation sociale, comme nous l'avons vu ci-dessus, le règlement de travail. Ainsi, le Chapitre IV de la loi de 1965 sur le règlement de travail est consacré aux pénalités qui peuvent être prévues par le règlement de travail. Ces dispositions touchent au pouvoir disciplinaire. Cette loi n'introduit pas de principe généralisé de "légalité " comme on le trouve en droit pénal. On pourrait donc croire que le principe nullum crimen, nulla poena sine lege ne s'applique pas.

Toutefois, la loi consacre un principe nullum crimen, nulla poena sine lege dans le chef de l'employeur qui veut infliger une amende. Ce principe n'est pas généralisé pour l'ensemble des pénalités. De plus, seules les pénalités prévues par le règlement de travail peuvent être appliquées. La loi ne fait expressément référence qu'à l'amende, mais d'autres sanctions disciplinaires peuvent être inscrites dans le règlement : le blâme, l'avertissement, la suspension temporaire de l'exécution du contrat de travail avec la perte de rémunération qui en résulte (mise à pied), etc. ${ }^{21}$. Ainsi, il est possible de conclure qu'une forme de principe généralisé Nulla poena sine lege est introduit.

La vigilance du législateur vis-à-vis des amendes s'explique par des usages abusifs tout au long du $19^{\text {ème }}$ siècle. Le produit des amendes n'est pas acquis à l'employeur : il doit être utilisé au profit des travailleurs de l'entreprise. Là où existe un conseil d'entreprise, la destination du produit des amendes doit être fixée en accord avec celui-ci22.

20 Article 15 e) de la loi portant organisation de l'économie, op. cit., et article 12 de la convention collective $n^{\circ} 9$ du 9 mars 1972 coordonnant les accords nationaux et les conventions collectives de travail relatifs aux conseils d'entreprise, http://www.cnt-nar.be/CCT-COORD/cct-009.pdf

21 La licéité de certaines sanctions disciplinaires telles que la mise à pied et la rétrogradation est contestée, au regard de la règle inscrite à l'article 25 de la loi sur les contrats de travail. Ne s'agitil pas, en effet, de clauses qui permettraient à l'employeur de modifier un élément essentiel du contrat de travail, ce qui est interdit par le droit belge ? Voy. sur cette question, V.Vannes, "Le droit disciplinaire dans l'entreprise », Revue de droit de I'U.L.B., 1991, p. 139 et spécialement, p. 154 et s. et F.Dorssemont, " De disciplinaire macht van de werkgever », in M. Rigaux, P.Humblet et G.Van Limberghen, Actuele Problemen van het arbeidsrecht 7, Antwerpen, 2005, p. 214-215.

22 Article 19 Loi du 8 avril 1965 instituant les règlements de travail. 


\section{3 - Le pouvoir de direction ou de gestion}

Le pouvoir de direction ou de gestion affecte le cœur même de la liberté d'entreprise et du droit de propriété des employeurs. En droit belge, une participation des travailleurs à ce pouvoir n'est pas formellement prévue. Les travailleurs ne participent pas formellement à la gouvernance de la société et ils ne sont pas associés à la direction ni à la gestion de l'entreprise ${ }^{23}$.

Si participation il y a, celle-ci se réduit à une sphère qui a trait à l'organisation du travail, elle ne concerne jamais l'orientation stratégique de l'entreprise. Tandis que les conséquences sociales de cette stratégie économique font l'objet d'un processus d'information et de consultation dès qu'il en résulte des projets de restructurations, la définition de la stratégie elle-même ne fait même pas l'objet d'un tel processus.

Il faudrait selon nous encore distinguer entre des pouvoirs de participation " aux décisions » et la participation à «la gestion ». Le pouvoir de gestion nous semble avoir plus de poids qu'un simple pouvoir de décision. Le pouvoir de gestion s'articule autour d'une multitude de décisions qui sont prises au quotidien. Un obstacle qui complique l'exercice de cette prérogative réside dans l'absence de personnalité juridique du conseil d'entreprise.

En termes de pouvoir de décision, on peut faire état de la compétence du conseil d'entreprise de faire le choix du bureau d'outplacement, lorsque la mission d'outplacement concerne un certain nombre de travailleurs; la décision est prise à la majorité des voix émises par la délégation patronale et à la majorité des voix émises par la délégation du personnel ${ }^{24}$. Cette implication des travailleurs doit être opérée lorsque l'employeur a I'intention de faire usage de l'outplacement dans son entreprise.

- la fixation de la date des vacances, pour autant qu'aucune décision n'ait été prise à ce sujet par la commission paritaire compétente ${ }^{25}$;

- la détermination du jour de remplacement d'un jour férié coïncidant avec un dimanche ou un jour habituel d'inactivité, pour autant qu'aucune décision n'ait été prise à ce sujet par la commission paritaire compétente ${ }^{26}$;

- l'établissement de la planification des congés-éducation payés ${ }^{27}$.

Ce pouvoir de décision dans le domaine de l'organisation du travail n'est pas réservé aux conseils d'entreprises. Dans des hypothèses bien déterminées, la délégation syndicale dispose aussi d'un pouvoir de décision. En Belgique, il y a une interdiction de mettre des travailleurs d'un employeur primus à la disposition d'un employeur secundus ${ }^{28}$. Il y a une exception principale qui concerne les travailleurs intérimaires. Le recours à des travailleurs intérimaires est encadré par des balises «objectives». Ce recours est autorisé dans un cas bien déterminé : celui du surcroît temporaire de travail. L'employeur ne peut invoquer

23 Ceci est évidemment à nuancer à partir de ce qui sera introduit dans la partie II

24 Convention collective n`51 du 10 février 1992 relative à l'outplacement, art. 6, http://www.cnt-nar. be/CCT-COORD/cct-051.pdf

25 A.R. 30 mars 1967 déterminant les modalités générales d'exécution des lois relatives aux vacances annuelles des travailleurs salariés, M.B., 6 avril 1967, art. 63.

26 Loi du 4 janvier 1974 relative aux jours fériés, M.B., 31 janvier 1974, art. 8.

27 Loi du 22 janvier 1985 de redressement contenant des dispositions sociales, M.B., 24 janvier 1985, art. 113.

28 Article 31 de la Loi du 24 juillet 1987 sur le travail temporaire, le travail intérimaire et la mise de travailleurs à disposition des travailleurs, M.B., 20 août 1987, p. 12495. 
cette condition que moyennant un accord préalable de la délégation syndicale ${ }^{29}$. Bien que la loi ne prévoit aucun recours contre un refus de la DS, dans un cas bien précis, le président du Tribunal de Hasselt a déclaré la requête unilatérale d'un employeur qui voulait obtenir une autorisation judiciaire se substituant à un tel refus, comme étant recevable et fondée $e^{30}$. En dehors d'un recours à des travailleurs intérimaires, la loi du 24 juillet 1987 permet aussi à un employeur qui n'est pas une agence intérimaire de mettre ses travailleurs à la disposition d'une autre entreprise à condition d'avoir l'autorisation d'un fonctionnaire ${ }^{31}$. La société utilisatrice doit, de son côté, chercher un accord avec la délégation syndicale en vue de pouvoir faire appel aux travailleurs mis à disposition. II est incontestable que ces prérogatives relèvent de la co-détermination.

Dans un domaine très spécifique les conseils d'entreprise disposent d'un véritable pouvoir de gestion.

L'article 15 h) de la loi portant organisation de l'économie (op. cit.) dispose que le conseil a la mission de « gérer toutes les œuvres sociales instituées par l'entreprise pour le bien-être du personnel ». Cette mission ne fait pas obstacle à la gestion autonome (non bipartite) de ces œuvres par les seuls travailleurs. Le Code du travail français évoque les activités sociales et culturelles ». L'article 13 de la CCT n 9 (op. cit) a précisé cette notion d'« œuvres sociales».

Il s'agit d'avantages répondant aux critères suivants : un caractère permanent ; être octroyé dans l'entreprise ; ayant pour objet de contribuer au bien-être des travailleurs de l'entreprise et ou des membres de leur famille; ne pas résulter d'une disposition légale ou réglementaire; ne pas être fixé par le contrat de travail ou d'emploi

La CCT $n^{\circ} 9$ énumère quelques exemples d'œuvres sociales : «les fonds et caisses d'entraide, les fonds de pension, les économats, les cantines et mess, les services de prêts et primes consentis par l'entreprise en vue de l'acquisition d'un logement, les activités récréatives et culturelles ».

Contrairement au conseil d'entreprise qui est appréhendé comme un organe essentiellement « passif » qui doit être informé et consulté, le CPPT participe à la politique et à la stratégie de l'entreprise dans le domaine spécifique du bien-être. Ici la prérogative du comité constitue un droit de participer à une stratégie d'entreprise afin d'identifier, de prévenir (éviter), d'évaluer et de limiter les risques relatifs à la santé et à la sécurité.

Cette mission est formulée en termes très larges. Le comité a pour mission d'émettre des avis et de formuler des propositions sur la politique de bien-être des travailleurs lors de l'exécution de leur travail ${ }^{32}$.

Ces prérogatives se matérialisent par des droits à l'information et à la consultation préalable. Exceptionnellement le comité dispose également d'un pouvoir de donner un accord préalable.

29 CCT n¹08 du 6 juillet 2013 relative au travail temporaire et au travail intérimaire, article 5, http:// www.cnt-nar.be/CCT-COORD/cct-108.pdf

30 Prés. trib. du Travail, Hasselt, 21 octobre 2009.

31 Article 32 de la Loi du 24 juillet 1987, op. cit.

32 Article 65 Loi du 4 août 1966 relative au bien-être des travailleurs lors de l'exécution de leur travail, M.B. 18 septembre 1996. 
Le comité est chargé d'émettre un avis préalable à la prise de décision dans certaines matières énumérées à l'article 3 de l'AR du 3 mai 199933. Cet avis préalable est requis sur:

$1^{\circ}$ tous les projets, mesures et moyens à mettre en œuvre qui, directement ou indirectement, immédiatement ou à terme, peuvent avoir des conséquences sur le bien-être des travailleurs lors de l'exécution de leur travail ;

$2^{\circ}$ la planification et l'introduction de nouvelles technologies en ce qui concerne les conséquences sur la sécurité et la santé des travailleurs, liées aux choix en matière d'équipements, de conditions de travail et à l'impact des facteurs ambiants au travail, à l'exception des conséquences auxquelles une convention collective de travail prévoyant des garanties équivalentes est d'application;

$3^{\circ}$ le choix ou le remplacement d'un service externe pour les contrôles techniques sur le lieu de travail et d'autres institutions ou d'experts;

$4^{\circ}$ le choix ou le remplacement des services auxquels il est fait appel en application des lois sur les accidents du travail;

$5^{\circ}$ toute mesure envisagée pour adapter les techniques et les conditions de travail à I'homme et pour prévenir la fatigue professionnelle;

$6^{\circ}$ les mesures spécifiques d'aménagement des lieux de travail afin de tenir compte, le cas échéant, des travailleurs handicapés occupés;

$7^{\circ}$ le choix, l'achat, l'entretien et l'utilisation des équipements de travail et des équipements de protection individuelle et collective.

Le comité doit encore donner son accord préalable sur la désignation, le remplacement ou la mise à l'écart des conseillers en prévention ${ }^{34}$. En cas de désaccord persistant, l'avis de l'inspecteur du travail compétent en matière de sécurité doit être sollicité (art. 20 §2) (5) $^{5}$.

Indépendamment de sa mission consultative, le comité peut prendre certaines initiatives dans des matières déterminées. Il peut ainsi jouer un rôle de promotion et exercer une action d'investigation et de contrôle (notamment art. 5, 6, 8 de l'AR du 3 mai 1999 précité). II peut ainsi :

a) élaborer et mettre en œuvre, dans les domaines qui lui sont propres, les moyens de propagande et les mesures concernant l'accueil des travailleurs, l'information et la formation à la prévention et la protection au travail ;

b) stimuler les activités du service interne et suivre le bon fonctionnement de ce service ;

c) examiner les plaintes formulées par les travailleurs en matière de bien-être au travail ainsi que les plaintes qui ont trait à la manière dont les services auxquels il est fait appel en application des lois sur les accidents du travail remplissent leur mission ;

d) élaborer des propositions visant à embellir les lieux de travail et leurs abords ;

e) participer à l'application du système dynamique de gestion des risques ;

33 Arrêté royal du 3 mai 1999 relatif aux missions et au fonctionnement des comités pour la prévention et la protection au travail, M.B., 10 juillet 1999.

34 Art. $20 \S 1$ de l'AR du 27 mars 1998 relatif au Service interne pour la prévention et la protection au travail M.B., 11 juin 1998.

35 En cas de licenciement, le préavis est nul ssil a été notifié avant que lıemployeur nıait demandé lsaccord du comité (Cour trav. Bruxelles, 25 février 1986, J.T.T., 1987, 170; R.W., 1986-1987, 582, note O.Van Achter 
f) désigner une délégation qui se rend immédiatement sur place, lorsqu'il y a des risques graves pour lesquels le dommage est imminent et chaque fois qu'un accident ou incident sérieux a eu lieu, ou lorsqu'un tiers au moins de la délégation des travailleurs au comité en fait la demande.

\section{II - LA PARTICIPATION DES TRAVAILLEURS AU NIVEAU DE LA SOCIÉTÉ EN DROIT (DES SOCIÉTÉS) BELGE}

En droit belge, la réglementation de la structure et du gouvernement de l'entreprise privée fait l'objet d'un corpus de règles spécifique : le droit des sociétés. Celui-ci a fait en 2017-2018 l'objet d'une réforme d'envergure destinée à transformer de manière conséquente le siège principal de la matière, à savoir le Code des sociétés, appelé à devenir un Code des sociétés et des associations ${ }^{36}$.

Bien que cette discipline juridique se saisisse au premier plan de la question de la répartition et de l'exercice du pouvoir de décision dans les entités économiques, à l'instar de ce qui a été montré dans la section précédente, les interactions entre le droit du travail et le droit des sociétés sont très limitées. Elles touchent notamment au rôle du réviseur d'entreprise, chargé de communiquer les informations économiques et financières relatives au fonctionnement de la société au conseil d'entreprise ${ }^{37}$ et plus largement, aux modalités de cette information financière ou encore à l'obligation de publier un bilan social, reprenant les aspects sociaux de ses activités ${ }^{38}$. Ces droits et obligations, qui ne représentent pas des prérogatives de participation dans le sens adopté plus haut, peuvent être envisagés comme contribuant à la démocratisation mentionnée en introduction, tout comme elles peuvent nourrir la négociation collective. Elles nourrissent parfois l'action d'un demos dans un sens plus étendu (le bilan social, comme les comptes annuels, sont rendus accessibles à tous les citoyens).

Traditionnellement, le régime de gestion des sociétés privées qui prévaut en droit belge est de nature moniste : coexistent ainsi pour chaque personne juridique une assemblée générale et un organe de direction (conseil d'administration dans les sociétés

36 Code des sociétés, adopté le 7 mai 1999, M.B., 6 août 1999, p. 29440. Entre autres changements substantiels, le code nouveau supprimerait la distinction entre sociétés civiles et commerciales, traiterait dans ses articles, tant du droit des sociétés que de celui des associations, autrefois séparés, limiterait le nombre de formes de sociétés à quatre (société en nom collectif, société à responsabilité limitée, société coopérative, société anonyme). Pour une présentation de la réforme en cours, nous renvoyons le lecteur au Précis de droit des sociétés 2018, à paraître très prochainement aux éditions Larcier. Pour un aperçu des travaux parlementaires en cours, voir le site de la Chambre des représentants:

https://www.lachambre.be/kvvcr/showpage.cfm?section=/none\&leftmenu=no\&language=fr\&cfm=/site/ wwwcfm/flwb/flwbn.cfm?lang=F\&legislat=54\&dossierlD=3119

37 Art. 151 et s. du Code des sociétés, op. cit. Le réviseur doit notamment « $3^{\circ}$ (...) analyser et d'expliquer à l'intention particulièrement des membres du conseil d'entreprise nommés par les travailleurs, les informations économiques et financières qui ont été transmises au conseil d'entreprise, quant à leur signification relative à la structure financière et à l'évolution de la situation financière de la société; (...) (Art. 151).

38 Loi du 22 décembre 1995 portant des mesures visant à l'exécution du plan pluriannuel pour l'emploi, M.B., 30 août 1995, p. 35333, Art. 44 et s. 
anonymes, gérants dans les SPRL) ${ }^{39}$. Une loi du 2 août $2002^{40}$, adoptée pour donner une première assise juridique aux principes issus de la corporate governance est toutefois venue permettre et encadrer la création d'un comité de direction dans les sociétés anonymes ${ }^{41}$. Ce comité de direction se voit déléguer certaines compétences par le CA, tandis que le conseil d'administration assure alors un rôle de supervision ${ }^{42}$. L'adoption de ce système facultatif ne constitue pas un vrai glissement vers le dualisme.

\section{A - UNE PLACE RESTREINTE POUR LES TRAVAILLEURS DANS LES ORGANES DE GESTION DE LA SOCIÉTÉ}

Très peu de dispositions du droit des sociétés visent l'implication des travailleurs salariés de l'entreprise dans sa gestion. Les associés disposent d'une grande liberté dans l'élaboration des statuts des sociétés. Ainsi, il est quasi toujours possible pour les travailleurs d'une société de participer à l'assemblée générale de celle-ci s'ils en détiennent du capital (pour autant qu'ils possèdent des actions assorties de droits de vote $)^{43}$, que cette acquisition soit explicitement prévue et parfois facilitée par les statuts (voy. infra), ou non ${ }^{44}$. De même, les statuts d'une société peuvent prévoir la représentation des salariés dans l'organe d'administration de la société, mais celle-ci n'est jamais imposée par le droit. Ce dernier cas se présente rarement en pratique. Les statuts peuvent encore prévoir des exclusions.

La question de la construction d'une démocratie économique, en particulier dans l'entreprise, est au cœur des revendications syndicales belges depuis la fin de la première guerre mondiale. Après la seconde guerre mondiale, et en dépit de désaccords importants entre les syndicats et le patronat, sa construction connut un moment important avec l'adoption de la loi du 20 septembre 1948 portant organisation de l'économie (voy.

39 Y. De Cordt (sous la dir.), Société anonyme, in Répertoire pratique du droit belge. Législation, Doctrine, Jurisprudence, Bruxelles, Bruylant, 2014, n 436.

40 Art. 33 de la loi du 2 août 2002 modifiant le Code des sociétés et la loi du 2 mars 1989 relative à la publicité des participations importantes, dans les sociétés cotées en bourse et réglementant les offres publiques d'acquisition, M.B., 22 aout 2002, p. 36555, introduisant un article 524bis dans le code des sociétés. La loi se fonde sur un rapport produit par la Commission gouvernementale sur la corporate governance: Commission gouvernementale de corporate governance, Naar een beter bestuur van de Belgische vennootschappen, Bruxelles, 20 mars 2000, http://www.law.ugent. be/econr/fli/WP/DeGrauwe.pdf, 35 p.

41 On retrouve cependant des discussions au Parlement belge autour d'une telle possibilité à la fin des années septante. A ce sujet : Commission Corporate Governance de I'Institut des Réviseurs d'Entreprise, Corporate Governance. La valeur ajoutée du réviseur d'entreprises, Rapport 2003, https://www.ibrire.be/fr/publications/series_cloturees/ibr_forum/Documents/2003/Commission_ corporate_governance.pdf pp. 4-6.

42 Art. 524bis du Code des sociétés: "(...) Si un comité de direction est institué, le conseil dıadministration est chargé de surveiller celui-ci. (...)». Contrairement aux solutions qui prévalent en Allemagne ou aux Pays-Bas, les membres du CA peuvent être membres du conseil de direction.

43 Dans les associations, tous les membres effectifs ont un droit de vote égal dans l'assemblée générale (Art. 7). Rien n'empêche les salariés de l'association d'être également membres de cellesci, pour autant que les fonctions qu'ils exercent en tant que membres ne remettent pas en question le lien d'autorité que I'ASBL exerce sur eux pour les fonctions salariées. Loi du 27 juin 1921 sur les associations sans but lucratif, les fondations, les partis politiques européens et les fondations politiques européennes, M.B., 1 juillet 1921, p. 5409.

44 Par ex. en application de l'article 609 du code des sociétés, op. cit. (augmentation du capital destinée au personnel), introduit par une loi du 18 juillet 1991. 
supra), instituant les conseils d'entreprise. Dans les années 1960-1970, la question de la participation des travailleurs aux organes de gestion des sociétés fut à nouveau l'objet de discussions, à la suite des événements de mai $1968^{45}$. Ces débats coïncident d'ailleurs dans le temps avec ceux qui touchaient aux modes de gestion de la future Societas Europaea ${ }^{46}$. Les deux grandes organisations syndicales belges, la Confédération des Syndicats Chrétiens (CSC-ACV) et la Fédération Générale des Travailleurs de Belgique (FGTB) partageaient alors un même idéal autogestionnaire : elles appelaient de leurs vœux la transformation des sociétés privées en des entités gérées par leurs seuls salariés. Dans les pratiques, elles offraient d'ailleurs leur soutien à certains travailleurs, confrontés à des fermetures d'entreprises de plus en plus nombreuses, dans la reprise de la gestion de celles-ci, au travers de la création d'ASBL et de coopératives. Ces expériences vécues d'autogestion restaient toutefois marginales.

Ces deux organisations partageaient également l'idée que la loi de 1948 était décevante : les compétences reconnues au conseil d'entreprise ne leur permettaient pas d'œuvrer à la démocratisation de l'entreprise qu'elles appelaient de leurs vœux. Sur le plan des idées, ces organisations ne parvenaient cependant pas à s'accorder sur les modalités de la transition vers l'autogestion, sur les dispositifs adéquats pour la faire advenir. En 1974, la CSC pousse un projet de remplacement des conseils d'entreprise par des «conseils de travailleurs», composés exclusivement de salariés, qui pourraient prendre des décisions de manière indépendante. La FGTB s'est opposée à cette idée et a plutôt plaidé pour un renforcement du contrôle ouvrier, via les canaux existants d'information et de revendication établis dans la loi et les conventions collectives (conseil d'entreprise et délégation syndicale). Ce syndicat était opposé à l'idée de confier des tâches de gestion patronale aux travailleurs. En l'absence de consensus entre ces organisations le cadre légal n'a pas été modifié dans ce sens. Les régimes d'information et de consultation du conseil d'entreprise ont cependant été renforcés, le réviseur d'entreprise s'est vu conférer un rôle pédagogique, et des règles contraignantes en matière de publication des comptes ont été adoptées, notamment en réponse à des revendications formulées par les syndicats, dans le but d'améliorer la qualité de l'implication des travailleurs dans la prise de décision.

L'adoption d'un Code de corporate governance en 2009 pouvait laisser présager une évolution des pratiques, puisque la prise en compte des parties prenantes dans le

45 Sur ce sujet passionnant, voy. Q. Jouan, «Syndicalisme chrétien et démocratisation de l'entreprise en Belgique. La bataille de l'information économique et financière ", Droit et travail, 2015, $n^{\circ} 7$, 14, p. ou L. Bettens, «La loi portant organisation de l'économie a 60 ans : regard rétrospectif sur les avancées du dialogue social mis en place... et ses limites ", Les analyses de I'IHOES, n47, 30 décembre 2008, http://www.ihoes.be/PDF/Ludo_Bettens-Elections_sociales_2.pdf, 17p. ; L. Ciccia, "Coopératives de travailleurs : le défi de l'autogestion pour les acteurs sociaux ", in SAW-B, Les coopératives. Un modèle tout terrien, Les dossiers de la SAW-B, 2011, pp. 104 à 125.

46 Voir pour un état de l'art des débats de l'époque: P. Kravaritou, « La convention collective de la société anonyme européenne », Cahiers de droit européen, 1971, pp. 284 à 305. Ce cadre ne fut finalisé qu'en 2004 par le Règlement (CE) n²157/2001 du Conseil du 8 octobre 2001 relatif au statut de la société européenne (SE), J.O.C.E., L 294, 10 novembre 2001, pp. 1-21. Le règlement est complété par une directive, qui accorde une flexibilité dans l'établissement des modalités de participation des travailleurs à la gestion, puisqu'il ouvre la possibilité à la création de SE dans lesquelles les travailleurs seraient représentés au sein des organes de direction, conformément au modèle allemand : Directive 2001/86/CE du Conseil du 8 octobre 2001 complétant le statut de la Société européenne pour ce qui concerne l'implication des travailleurs, J.O., L 294, 10 novembre 2001, pp. 22 à 32. 
gouvernement des entreprises est l'un des objectifs visés par ce discours et ses partisans. Le Code limite cependant l'ouverture des organes de gestion aux femmes et à la "diversité ", sans évoquer la situation des salariés : " 2.1 La composition du conseil d'administration assure que les décisions soient prises dans l'intérêt social. Cette composition est basée sur la mixité des genres, et la diversité en général, ainsi que sur la complémentarité de compétences, d'expériences et de connaissances. Une liste des membres du conseil d'administration est publiée dans la Déclaration GE ${ }^{47}$. Ce Code n'a pas de valeur juridique contraignante ; il se présente comme un guide à destination des dirigeants des sociétés cotées ${ }^{48}$.

\section{B - Quelques tRACES DE PARTICIPATION DES TRAVAILLEURS}

En dépit de ce contexte peu favorable, on retrouve tout de même des traces de participation des salariés à la gestion de l'entreprise, au cœur du droit des sociétés belges.

\section{1 - Participation financière}

Dans les années 1990 ont émergé des pratiques volontaires de participation des travailleurs au capital et aux bénéfices des sociétés. Ces possibilités existent toujours mais une formule spécifique a été instaurée par une loi du 22 mai 2001 relative aux régimes de participation des travailleurs au capital et aux bénéfices des sociétés ${ }^{49}$. Parmi les traits intéressants du régime se trouve sa voie d'instauration : la participation financière est organisée dans chaque entreprise qui le souhaite (il s'agit donc d'un régime facultatif) au travers de l'élaboration, par voie de concertation sociale, d'un plan de participation entre l'employeur et les représentants de ses travailleurs. Ce régime peut passer par la création d'une "société coopérative de participation", "ayant pour objet exclusif de détenir et de gérer les participations au capital apportées par les travailleurs $»^{50}$. La concentration des parts des travailleurs au sein d'une telle société peut accroître le pouvoir de contrôle des travailleurs.

47 Code de corporate gouvernance, adopté le 12 mars 2009 par la Commission Corporate Governance, https://www.corporategovernancecommittee.be/sites/default/files/generated/files/ page/corporategovfrcode2009.pdf, principe 2. Ce code révise une version antérieure, adoptée en 2004, afin de répondre aux « nouvelles aspirations de la société civile et des parties prenantes (stakeholders) quant aux responsabilités et à la conduite des sociétés, des administrateurs et des managers. » (p. 4)

48 Y. De Cordt, «Introduction », in Droit des sociétés - Millésime 2011, Bruxelles, Larcier, 2011, pp. 7-8, n 5.

49 Loi du 22 mai 2001 relative à la participation des travailleurs au capital des sociétés et à l'établissement d'une prime bénéficiaire pour les travailleurs, M.B., 9 juin 2001, p. 192888. Parmi les nombreux ouvrages consacrés à cette question, à laquelle nous ne consacrons que quelques lignes, voir en particulier, La participation des travailleurs au capital et aux bénéfices des sociétés. Commentaires de la loi du 22 mai 2001, Actes d'un colloque organisé par le département de droit économique et social de I'UCLouvain, Bruxelles, Bruylant, 2001 ; La participation financière des travailleurs, Actes d'un colloque organisé par le département de droit économique et social de I'UCLouvain, Bruxelles, Bruylant, 1998 ; M. Goldfays et J.N. Letambert, Plans de participations financières. Travailleurs capitalistes : la fin d'une antinomie? Waterloo, Kluwer, coll. Etudes pratiques de droit social, n 7, 2002 ; P. Marchandise, "Salarié et actionnaire. Récit et réflexions d'un juriste d'entreprise à propos des augmentations de capital réservées au personnel ", in L'entreprise te ses salariés : quel partenariat? Séminaire organisé à Liège le 22 octobre 2009, Bruxelles, Bruylant, 2009, pp. 371 à 392 ; J.Malherbe et A. de Schoutheete, "La participation des travailleurs au capital et aux bénéfices des sociétés », J.T., 10 mai 2003 ; n6096, pp. 345 à 356.

50 Art. 12 et s., Loi du 22 mai 2001. 
L'adoption de ce régime a été présentée par certains spécialistes du droit des sociétés, tenant d'une approche "stakeholderiste " de la corporate governance, comme un pas ambitieux vers la démocratisation dans la doctrine belge de droit des sociétés, puisque la " participation » était enfin reconnue aux travailleurs. Si ceux-ci ne se voyaient pas donner des actions en échange de leur " apport en travail », les plans de participation financière leur permettent d'acquérir une part des bénéfices, ou ces actions-mêmes, à titre gratuit ou à prix réduit, au titre de rémunération. A ces titres sont le plus souvent assortis des droits patrimoniaux mais aussi politiques, même si leur effectivité dépend de la puissance votale des salariés ${ }^{51}$.

Cependant, il s'est surtout agi de vœux pieux. Le régime est réservé à un public très spécifique de travailleurs, surtout des cadres dirigeants. Les parts octroyées aux travailleurs ne sont pas toujours assorties de droit de vote. Souvent, elles constituent une forme de rémunération alternative, accordée aux travailleurs à condition qu'ils remplissent certains objectifs de production ${ }^{52}$. Certains parlent de régime lacunaire. La raison d'être de la réforme peut être radicalement interrogée: démocratisation du gouvernement de l'entreprise, y compris fondée sur un nouveau partage de la propriété et/ou de la richesse créée dans la société? Ou plutôt création d'incitants à accroître encore davantage la productivité, sans modifier les rapports hiérarchiques dans le conduit des affaires de l'entreprise ?53

\section{Sociétés à finalité sociale}

En 1995 fut introduit en droit belge un cadre juridique visant la société à finalité sociale. ${ }^{54}$ L'article 661 du Code des sociétés, introduit par la loi du 13 avril 1995, dispose que « les sociétés dotées de la personnalité juridique énumérées à l'article $2, \S 2{ }^{55}$, [à l'exception des sociétés européennes] [et des sociétés coopératives européennes, ] sont appelées sociétés à finalité sociale lorsqu'elles ne sont pas vouées à l'enrichissement de leurs associés (...)». Le régime ne créée pas une nouvelle forme de société, mais représente une option offerte à toute société qui souhaite poursuivre des activités commerciales ou industrielles et une finalité sociale, précisée dans ses statuts.

A l'époque, des débats avaient émergé, qui portaient à nouveau sur l'opportunité d'introduire une représentation du personnel dans les organes d'administration de ces

51 Par exemple, J. Malherbe etY. de Cordt, «La participation des travailleurs au capital et aux bénéfices des sociétés. Aspects de droit des sociétés », in X. La participation des travailleurs., 2001, op. cit., pp. 206 à 208.

52 L'objectif de participation n'est pas repris dans la liste des objectifs poursuivis par les plans de participations financières identifiés par S. Stenuick, Les bonus, Waterloo, WoltersKluwer, coll. Entreprise et droit social, 2017, p. 26.

53 Dans ce sens, A. Dewandre, "La participation des travailleurs à la gestion. Prolonger la réflexion », Analyse de la SAW-B ASBL, Septembre 2009, http://www.saw-b.be/EP/2009/A0910.pdf, pp. 4 à 6.

54 Sur ce sujet, P. Nicaise, "Les sociétés à finalité sociale et la participation des travailleurs ", in La participation financière, 1998, op. cit., pp. 187 et s. M. Stroobant, « De venootschap met een sociaal oogmerk », in De gewijzigde venootschapswet 1995, Anvers, Kluwer, 1996, pp. 85 à 100 ; M. Coipel, "Les alternatives à l'ASBL pour l'exercice d'activités marchandes dans une optique non lucrative », in M. Coipel et M. Marée (coord.), ASBL et activités marchandes, Les Dossiers d'ASBL Actualités, Liège, Edipro, 2009, n8, pp. 109 à 127 ; D. D’Hulstere et J.P. Pollenus, La société à finalité sociale, en questions... et en réponses ! Liège, Edipro, 2008.

55 C'est-à-dire les sociétés suivantes: sociétés en nom collectif, en commandite simple, à responsabilité limitée, coopérative, anonyme, en commandite par actions, le groupement d'intérêt économique. 
sociétés particulières. Leur nature spécifique -d'être constituées dans le but de poursuivre des finalités d'intérêt général- aurait peut-être justifié un tel ajout. Comme en témoigne cet extrait, aucun accord ne putêtre trouvé, la proposition ne recueillant même pas l'assentiment des syndicats belges : "En ce qui concerne le conseil d'administration, tant la FGTB que la CSC estiment que le problème relève de la compétence du Conseil national du travail. Ces organisations ne soutiennent d'ailleurs pas une telle innovation. La participation peut toujours être organisée par les statuts, mais elle ne peut être obligatoire $»^{56}$.

Plusieurs traits de la société à finalité sociale vont cependant dans le sens d'une démocratisation de la gestion des entreprises dites " d'économie sociale », objectif porté par les partisans de la loi. Elles concernent leur participation au capital et leur puissance votale à l'assemblée générale. Ainsi, ces sociétés ne peuvent être considérées comme telles que "lorsque leurs statuts: (...) $4^{\circ}$ stipulent que nul ne peut prendre part au vote à l'assemblée générale pour un nombre de voix dépassant le dixième des voix attachées aux parts ou actions représentées; ce pourcentage est porté au vingtième lorsqu'un ou plusieurs associés ont la qualité de membre du personnel engagé par la société; $(\ldots) 7^{\circ}$ prévoient les modalités permettant à chaque membre du personnel d'acquérir, au plus tard un an après son engagement par la société, la qualité d'associé; cette disposition ne s'applique pas aux membres du personnel qui ne jouissent pas de la pleine capacité civile ; $8^{\circ}$ prévoient les modalités permettant que le membre du personnel qui cesse d'être dans les liens d'un contrat de travail avec la société perde, un an au plus tard après la fin de ce lien contractuel, la qualité d'associé (...) $\Perp^{57}$. La participation des travailleurs est donc un droit qui doit être offert aux travailleurs dans ces sociétés.

La société à finalité sociale ne doit pas être confondue avec la société coopérative, elle aussi réglementée par le code des sociétés, dont les origines remontent au 19 ème $^{\text {siècle }}{ }^{58}$. La société coopérative était historiquement censée offrir le cadre le plus propice à des sociétaires désireux de faire prospérer un projet d'intérêt collectif, visant les intérêts des membres. Le cadre réglementaire très souple (capital de départ réduit, grande liberté statutaire) a séduit de plus en plus d'entrepreneurs, dont les intentions ne cadraient pas avec les visées d'origine, à partir des années 1980. Aujourd'hui, seule une partie réduite des coopératives fonctionnent en respect des principes du mouvement coopératif des origines (solidarité, entraide, démocratie). A condition de remplir certaines conditions, elles peuvent obtenir un agrément délivré par un organisme public ad hoc, le Conseil national de la coopération ${ }^{59}$ : cette option, ouverte depuis 1955, permet un tri entre les coopératives. Cet agrément leur procure des avantages sociaux et fiscaux. Dans la réforme du code des sociétés mentionnée plus haut, il est prévu que ce régime soit fortement modifié : seule la société coopérative pourra adopter une finalité sociale, moyennant l'obtention par elle d'un agrément ${ }^{60}$.

56 Doc. Parl., Sénat, 1993-1994, n 1086-2, p. 267

57 Art. 661 du Code des sociétés, op. cit. Notez qu'un projet de loi introduit en 2007 visait à renforcer ces modalités de participation. II n'a pas abouti.

58 Code des sociétés, op. cit., Livre VII, articles 350 et s. Sur cette question, voir M. Vanhove, "Les coopératives en Belgique ", in SAW-B, op. cit., pp. 44 à 63.

59 Site officiel : http://newsletter-nrc-cnc.be/index.php/fr/. En 2008, 1 \% des coopératives étaient agréées: M. Vanhove, op. cit., p. 57.

60 Voir la critique formulée par le Groupe Terre, «L'entreprise à finalité sociale mise au placard », Le Soir, 28 juin 2018, http://plus.lesoir.be/165083/article/2018-06-28/lentreprise-finalite-sociale-mise-au-placard. 


\section{Le dirigeant-salarié}

En théorie, les directeurs de sociétés commerciales sont susceptibles d'être révoqués ad nutum par l'assemblée générale des actionnaires, dont ils ont reçu le mandat de gestion. Pour cette raison, ceux-ci ne peuvent être employés sous contrat de travail, car le statut de salarié requerrait de leurs employeurs qu'ils respectent, pour les licencier, les règles fixées dans la loi du 3 juillet 1978. Par ailleurs, les dirigeants ne se trouvent généralement pas dans une situation de subordination juridique. L'administrateur et le gérant sont donc le plus souvent des travailleurs indépendants.

Depuis les années 1980 cependant, le recours à des dirigeants-salariés a été accepté par la doctrine. Les juges ont apporté leur soutien à ces pratiques volontaires. Pour qu'un dirigeant de société puisse être employé sous contrat de travail cependant, il faudra que l'existence d'une autorité subie par lui soit démontrée pour au moins une partie de ses tâches ${ }^{61}$. Le cas échéant, il pourra cumuler un contrat de travail avec un mandat de représentation exercé en tant qu'indépendant. La jurisprudence nous enseigne toutefois que l'autorité ne doit pas être exercée effectivement; il suffit qu'elle soit possible ${ }^{62}$. Aujourd'hui, il n'existe toutefois pas de régime homogène encadrant le recours à la figure de dirigeant-salarié, ce qui créée une certaine insécurité juridique. Les solutions varient en fonction du type de société concernée et surtout, du type de fonction dirigeante exercée (administrateur, délégué à la gestion journalière, administrateur délégué, membre du comité de direction, gérant statutaire ou non).

L'administrateur-salarié est un travailleur salarié qui occupe un poste clé dans le gouvernement de l'entreprise. Cependant, cette participation ne s'étend pas au-delà de lui seul. On peut aussi émettre des doutes quant à l'usage que ce salarié particulier fera des droits qu'il tire, tant du droit des sociétés que du droit du travail. II est peu probable que celui-ci soit amené à défendre dans ses fonctions de gestion, la collectivité des travailleurs de l'entreprise, en particulier contre la direction de la société, que bien souvent il incarne ou représente. Cette ouverture a surtout contribué à accorder une plus grande stabilité d'emploi à certains dirigeants d'entreprise.

\section{UN CAS EXCEPTIONNEL : LA SNCB}

Le cas de la SNCB constitue une exception ambitieuse aux modalités générales de représentationcollectivedestravailleursdanslesentreprises, tantpubliquesqueprivées ${ }^{63}$.Cela tientaux modalités ambitieuses de la concertation sociale, organisée au sein de «commissions paritaires ». Le gouvernement de cette société fut également caractérisé par la présence d'administrateurs salariés au sein du conseil d'administration, depuis sa fondation en 1926.

61 Sur ce sujet épineux qui fait l'objet d'une littérature abondante, voir notamment F. Kefer, « Les salariés impliqués dans les organes de direction d'une société commerciale », J.T.T., 10 décembre 2009, p. 433 ; A. Gillet et M. Michaud-Nerard, "Le statut social du dirigeant d'entreprise : un point sur la question », Orientations, Décembre 2010, n²10, p. 1.

62 Par ex. : Cass. 13 juin 1968, Arr. Cass., 1968, p. 1239 ; Cass. 15 février 1982, R.W., 1982-1983, p. 2210 ; Cass. 4 février 2013, J.T.T., 2013, p. 201.

63 Sur ce sujet, le lecteur consultera : D. Luyten, "La SNCB en tant qu'employeur », in B. Van der Herten, M. Van Meerten et G. Verbeurgt, Le temps du train. 175 ans de chemins de fer en Belgique, 2001, Louvain, Leuven University Press, pp. 274 à 300 ; D. Nuchelmans et G. Pagano, « Les entreprises publiques autonomes », Courrier Hebdomadaire du CRISP, 1991, n¹321-1322, p. 50. 
La règle fut fixée à l'article $7,4^{\circ}$ de la loi du 26 juillet 1926 : «Trois membres (du Conseil d'administration) sont nommés par le personnel ${ }^{64}$. A l'époque, le principe de cette représentation faisait l'objet d'un consensus au sein du Parlement, tout comme les modalités de désignation, non précisées dans la loi : « Je vois avec plaisir que le principe de la représentation du personnel dans le Conseil d'administration ne semble être contesté par aucun des groupes politiques de cette Chambre. La seule difficulté est de savoir comment la représentation du personnel sera réalisée. On peut la concevoir de deux façons: ou bien une représentation des organisations syndicales, ou bien une représentation résultant du scrutin de tous les agents organisés en collèges électoraux. Ce second mode de désignation entraînera un système d'élections fort compliqué. Après examen, c'est la première manière que nous avons adoptée... $»^{65}$.

Par la suite, la structure et l'administration de la SNCB ont plusieurs fois évolué. En 1986, le nombre d'administrateurs a été réduit de vingt-et-un à seize et ceux désignés par les organisations syndicales au nombre de deux $x^{66}$. En 1991, une loi sur les entreprises publiques autonomes fut adoptée afin d'établir un nouveau statut pour certaines grandes entreprises publiques (régie des télégraphes et des téléphones, des postes, des voies aériennes et la SNCB), confrontées à une concurrence grandissante, en évitant d'avoir recours à la privatisation, « qui permette la sauvegarde de sa mission sociale tout en limitant le financement public d'activités commerciales pour lesquelles le monopole public ne se justifie plus ${ }^{67}$. La situation spécifique de la SNCB, intégrée à la loi sur les entreprises publiques autonomes, a pu être conservée avec l'adoption de cette loi, mais elle n'a par contre pas pu être élargie aux autres entreprises publiques autonomes.

En 2002, le gouvernement adapte encore les structures de la SNCB ${ }^{68}$. II crée un " comité stratégique » au sein du CA, aux compétences limitées, dans lequel siègent six membres représentant les organisations représentatives des travailleurs. Ceux-ci ne sont par contre plus représentés au Conseil d'administration lui-même. En 2004, la SNCB est scindée en trois entités, pour répondre à des exigences européennes : la SNCB-Holding, qui chapeaute et met à disposition du personnel pour Infrabel et la "nouvelle SNCB", transformée en société anonyme de droit public ${ }^{69}$. Toutes trois se sont vues reconnaître le statut d'entreprise publiques autonomes. C'est la SNCB-Holding qui a hérité du comité stratégique dans sa structure organique.

64 Art. 7, 4', Loi du 26 juillet 1926 relative à la SNCB et au personnel des chemins de fer belges, M.B., 24 juillet 1926, p. 3892. (version d'origine)

65 Rapport de M. Brusselmans, Ann. Parl., Ch. repr., 1925-1926, p. 2298. Et aussi : « La Section centrale entend que les membres du Conseil d'administration à désigner par le personnel, le seront par les organisations syndicales, comme la pratique existe déjà au Département des chemins de fer. " (Rapport à la Chambre, Doc. Parl., Ch. repr., 1925-1926, n² 418, p. 1926), cités par P. Dirickx, «L'acte de naissance de la SNCB : la loi du 23 juillet 1926 », Le Rail, Juillet 1976, reproduit sur http://rixke. tassignon.be/spip.php?article1414

66 A.R. du 29 août 1986 modifiant la loi du 23 juillet 1926 créant la Société Nationale des Chemins de fer belges, M.B., 16 septembre 1986, p. 12537.

67 D. Nucherlmans et G. Pagano, op. cit., p. 8.

68 Loi du 22 mars 2002 portant modification de la loi du 21 mars 1991 portant réforme de certaines entreprises publiques économiques, M.B., 26 mars 2002, p. 12554.

69 A.R. du 14 juin 2004 portant réforme des structures de gestion de l'infrastructure ferroviaire, M.B., 24 juin 2004. et A.R. du 18 octobre 2004 portant certaines mesures de réorganisation de la Société nationale des Chemins de fer belges, M.B., 20 octobre 2004, 
En 2013, la SNCB a subi une dernière réforme, passant d'une structure tricéphale à une autre, bicéphale, avec deux entreprises : la SNCB (entreprise ferroviaire) et Infrabel (gestionnaire de réseau) $^{70}$. Une nouvelle structure HR Rail, filiale des deux entreprises, est chargée de recruter le personnel qu'elle mettra à disposition des deux entités. Le comité stratégique de la SNCB-Holding est supprimé et remplacé par un « comité d'entreprise stratégique ", dans les deux nouvelles entités principales ${ }^{71}$. Ses compétences font plus de lui un organe de concertation sociale similaire à un conseil d'entreprise qu'un organe de gestion de l'entreprise ${ }^{72}$. A la cogestion des origines se sont donc substituées des modalités classiques d'implication des travailleurs (information et consultation). II convient toutefois de mentionner l'existence d'une modalité extraordinaire et très forte de co-détermination (dans le sens introduit dans la partie I de cette contribution). Le conseil d'administration ne saurait arrêter des modifications du «statut syndical » et du « statut du personnel » que moyennant un vote préalable et positif de la majorité des deux tiers des voix, exprimées au sein de la commission paritaire nationale, l'instance principale de représentations des travailleurs au sein de la SNCB. Ce vote lie d'ailleurs le conseil d'administration de la SNCB ${ }^{73}$

\section{CONCLUSION : LA PARTICIPATION RETROUVÉE ?}

Cette brève étude a été consacrée à la participation des travailleurs salariés au gouvernement des entités économiques et sociales dans lesquelles ils exercent leurs activités, telle que consacrée dans le droit belge. En droit du travail, les prérogatives de participation ont été rangées en trois catégories : participation au pouvoir réglementaire, disciplinaire et de gestion/de direction. Il a été montré que ces prérogatives occupaient un rôle marginal, en droit belge. A l'exception du pouvoir réglementaire les travailleurs sont le plus souvent tenus en marge de l'exercice du pouvoir, seules des prérogatives d'information et de consultation leur sont alors reconnues. Ce n'est pas tant au travers d'une " participation » stricte, mais plutôt de l'action syndicale, exercée dans l'entreprise droit de négociation et d'action collective surtout - que les travailleurs peuvent influer sur le gouvernement de l'entreprise. En droit des sociétés, il n'est guère trouvé de manifestation plus ambitieuse de participation des travailleurs. Seules existent quelques traces de participation : participation financière, reconnaissance du statut de salarié à certains directeurs, quelques exemples de sociétés dont le cadre réglementaire ou les statuts aménagent une place aux salariés dans leurs organes de décision. Cette conclusion un peu tiède nous pousse toutefois à quelques constats supplémentaires.

D'une part, il peut exister un écart entre le droit et les pratiques développées par les acteurs. C'est dans la nature-même du discours juridique de présenter des interstices dont peuvent profiter les acteurs pour déployer leur créativité. La société SMartCoop est un excellent exemple d'une telle attitude ${ }^{74}$. Dans ses statuts, la nouvelle SMartCoop

70 Loi du 30 août 2013 relative à la réforme des chemins de fer belges, M.B., 13 septembre 2013, et l'arrêté royal qui met en œuvre la réforme du cadre normatif : A.R. du 11 décembre 2013 portant réforme des structures de la SNCB Holding, d'Infrabel et de la SNCB, M.B., 16 décembre 2013.

71 Art. 127 à 131 de la loi du 23 juillet 1926, op. cit.

72 J. Delvoie et M. Heine, «La réforme des chemins de fer belges du 1 er janvier 2014 : aperçu de I'architecture juridique », R.D.I.R., 2014/3, p. 302.

73 Article 75 Loi du 23 juillet 1926 relative à la SNCB et au personnel des Chemins de fer belges

74 Voir pour une présentation du projet de l'organisation : http://smartbe.be/fr/la-cooperative-enpratique/ 
(anciennement ASBL SMart) prévoit expressément la participation de ses salariés - tant ses salariés permanents que ses "membres-salariés ", qui développent des projets professionnels autonomes avec le soutien de l'organisation - à l'assemblée générale. Tous sont ou incités à acquérir une part de la coopérative. Les membres sont regroupés dans une catégorie $A$ de sociétaires, qui leur est réservée en exclusivité ; les permanents sont intégrés à la catégorie par défaut, B (pouvant comprendre toute personne intéressée par le projet). Le conseil d'administration ne se compose que de sociétaires, dont $3 / 5^{\text {ème }}$ appartiennent à la catégorie $\mathrm{A}$ et $2 / 5^{\text {ème }}$ appartiennent à la catégorie $\mathrm{B}^{75}$.

L'actualité nous montre les limites des dispositifs légaux de participation : on pense récemment au cas de la fermeture de l'entreprise Caterpillar à Gosselies. Certains syndicats, représentants politiques et experts dénoncent les insuffisances des procédures d'information et de consultation prévues dans des situations de restructuration, surtout lorsque les prises de décisions ont lieu en dehors du territoire belge. Un débat est en cours au sein du Conseil National du Travail ${ }^{76}$. La situation de Caterpillar est d'autant plus emblématique que des nouveaux dispositifs de " participation " avaient justement été expérimentés dans l'entreprise. Ceux-ci portaient essentiellement sur l'implication des travailleurs dans l'adaptation des tâches ${ }^{77}$.

Cette courte étude montre que le débat autour d'un renforcement de la participation des travailleurs dans la gestion de l'entreprise est récurrent et transversal. L'une des raisons des échecs répétés de solutions ambitieuses, en plus des désaccords politiques et professionnels, tient au cloisonnement des disciplines, du droit social et du droit des sociétés, pourtant tous deux préoccupés par la question de la direction de l'entreprise. Nous ne pouvons que souhaiter que le dialogue entre ces champs du droit s'accentue, à l'image de ce qui se produit actuellement en France autour de la loi PACTE et du rapport Notat-Senard, remis en 2018.

Plusieurs initiatives législatives belges récentes vont dans le sens de la promotion d'une plus grande participation des travailleurs. Parmi elle, nous rappelons la proposition de loi modifiant la loi du 20 septembre 1948 (...) visant à créer un droit d'alerte économique et social au sein des conseils d'entreprise ${ }^{78}$; la proposition de loi visant la création d'un statut pour les sociétés à gestion coopérative et participative ${ }^{79}$; la proposition de loi (...) en ce qui concerne les droits des délégués du personnel au sein des conseils d'entreprise et le droit

75 Voir les statuts de SMartCoop, en particulier les articles 9, 22 et 37 al. 3 et $4:$ http://smartbe.be/ media/uploads/2017/01/Statuts-SMart-Coop-FR.pdf

76 Pour un énoncé sommaire de ces critiques, mentionnant le cas de Caterpillar :O.Scheuer et F. Delchevalerie, "La loi Renault : 20 ans après », Lalibre.be, 25 mars 2017, http://www.lalibre.be/ economie/libre-entreprise/la-loi-renault-20-ans-apres-58d295fccd70a15c9a4e0ddd; N. Bamps,

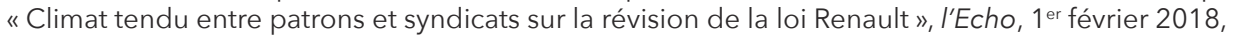
https://www.lecho.be/economie-politique/belgique/federal/climat-tendu-entre-patrons-etsyndicats-sur-la revision-de-la-loi-renault/9977949.html

77 Pour une analyse éclairante des modalités de participation proposées aux travailleurs de l'usine Caterpillar à Gosselies, voir J. Charles, La participation en actes. Entreprise, ville, association, Paris, Desclée de Brouwer, coll. «Solidarité et société », 2016, 168 p

78 Proposition de loi modifiant la loi du 20 septembre 1948 portant organisation de l'économie, visant à créer un droit d'alerte économique et social au sein des conseils d'entreprise, Ch., 20162017, n² 2146/001. (introduite par des parlementaires d'Ecolo-Groen, le 9 Novembre 2016).

79 Proposition de loi visant la création d'un statut pour les sociétés à gestion coopérative et participative, Ch., 2014-2015, n 1068/001. (Déposée par le parti Ecolo-Groen, le 6 mai 2015). 


\section{LA PARTICIPATION DES TRAVAILLEURS EN DROIT BELGE}

d'alerte conféré au $\mathrm{CE}^{80}$; la proposition de loi modifiant le code des sociétés, concernant la représentation des travailleurs au sein du comité de rémunération ${ }^{81}$, ou encore la proposition de loi visant à permettre l'information du Parlement en cas de licenciement collectif dans une entreprise ${ }^{82}$.

80 Proposition de loi modifiant la loi du 20 septembre 1948 portant organisation de l'économie en ce qui concerne les droits des délégués du personnel au sein des conseils d'entreprise, Ch., 2014, $n^{\circ}$ 0349/001, (déposé par le parti PS, le $1^{\text {er }}$ Octobre 2014).

81 Proposition de loi modifiant le Code des sociétés, concernant la représentation des travailleurs au sein du comité de rémunération, Ch., 2014, n0161/001 (déposée par le parti Défi le 26 Août 2014).

82 Proposition de loi visant à permettre l'information du Parlement en cas de licenciement collectif dans une entreprise, Ch., 2015-2016, n²036/001 (déposée le 15 septembre 2016 par le parti Ecolo-Groen).

\section{FILIP DORSSEMONT}

Professeur de droit du travail, Université Catholique de Louvain, Crides .

Thèmes de recherche : Droit des relations collectives- droits fondamentaux des travailleursdroits fondamentaux au travail-droit du travail de I'Union européenne-droit du travail et religion.

\section{Publications :}

$\sim$ F. Dorssemont, « Worker's rights to information and consultation within the undertaking ", in S. Peers et alii, The EU Charter of fundamental rights, Hart Publishing, Oxford, 2014, p.749.

$\sim$ F. Dorssemont, Th. Blanke, The Recast of the European Works Council Directive, Antwerpen, Intersentia, 2010, 410 p.

$\sim$ F. Dorssemont, « The right to information and consultation in article 27 of Charter of Fundamental rights of the European union : les than a right and less than a principle just an ordinary provision lacking direct effect ?», Maastricht Journal of European and Comparative Law, Vol. 2014, p. 704.

\section{AURIANE LAMINE}

Professeure de droit du travail, Université Catholique de Louvain, Crides.

Thèmes de recherche : Droit des relations collectives, droits fondamentaux des travailleurs, droits fondamentaux au travail-droit du travail de l'Union européenne-droit du travail et religion.

\section{Publications :}

A. Lamine et J. Prassl, "Collective autonomy for on-demand workers? Normative arguments, current practices and legal ways forward", in S. Laulom et F. Hendrickx (éds.), Collective bargaining developments in times of crisis, Bulletin of Comparative Labour Relations, Wolters Kluwer, 2018, p. 269.

A. Lamine, "Les droits des travailleurs en cas de transfert d'une entreprise insolvable", Journal de droit européen (J.D.E.), Mai 2018, n²49, p. 166.

A. Lamine, "Articulation des normes et protection du travailleur. Quelques réflexions à partir des Accords d'entreprise transnationaux", Revue de droit social (R.D.S.), numéro spécial "aux sources du droit social", 2017, p. 433. 